\title{
Characterization of Salmonella enterica serovar Typhimurium and Salmonella enterica serovar 4,[5],12:i:- isolates from pigs presenting with diarrhea in Korea
}

\author{
Ki-Eun LEE ${ }^{1}$, Deog-Yong LEE ${ }^{2)}$, Hwan-Won CHOI ${ }^{3)}$, Su-Jin CHAE'), Young-Sun YUN ${ }^{2)}$, Ki-Chan LEE ${ }^{1)}$, \\ Yun-Sang $\mathrm{CHO}^{1)}$ and Dong-Kun YANG ${ }^{1) *}$ \\ 1) Animal and Plant Quarantine Agency, Anyang 430-757, Republic of Korea \\ ${ }^{2)}$ Korea National Institute of Health, Cheongju 363-951, Republic of Korea \\ ${ }^{3)}$ ChoongAng Vaccine Laboratories, Daejon 305-348, Republic of Korea
}

(Received 17 March 2015/Accepted 27 May 2015/Published online in J-STAGE 14 June 2015)

ABSTRACT: Between 2011 and 2012, a total of 896 pig fecal samples were collected from nine provinces in Korea, and 50 salmonella enterica susp. enterica serovar Typhimurium ( $S$. Typhimurium) was isolated. The characteristics of the 50 strains were analyzed, and 4 strains were identified as Salmonella enterica subsp. enterica serovar 4,[5],12:i:-. Salmonella 4,[5],12:i:- could not be distinguished from $S$. Typhimurium through phage typing, antimicrobial resistance testing or multiple-locus variable-number tandem repeat analysis (MLVA). However, among the four Salmonella 4,[5],12:i:- strains, one (KVCC-BA1400078) was identified as a Salmonella 4,[5],12:i:- clone isolated from humans in the United States, and another (KVCC-BA1400080) was identified as DT193, which has been primarily isolated from humans and animals in European countries. The presence of Salmonella 4,[5],12:i:- in Korea poses a significant threat of horizontal transfer between pigs and humans.

KEY WORDS: Korea, Salmonella 4,[5],12:i:-, S. Typhimurium, swine

doi: 10.1292/jvms.15-0151; J. Vet. Med. Sci. 77(11): 1511-1515, 2015

Salmonella spp. are important zoonotic pathogens commonly found in farmed animals. In pigs, salmonellosis is an infectious digestive disease that presents with acute or chronic symptoms. In surveys of Salmonella in pigs, Salmonella enterica subsp. enterica serovar Typhimurium $(S$. Typhimurium) and $S$. Derby are commonly isolated worldwide, whereas the host-adapted $S$. Choleraesuis is now rare in Europe and Australia but still frequently found in North America and Asia. In Korea, $S$. Typhimurium is the main cause of digestive disease in pigs $[4,25]$.

Recently, there have been many reports of $S$. Typhimurium variants isolated from humans and warm-blooded animals [13]. Among these variants, Salmonella enterica subsp. enterica serovar 4,[5],12:i:- (Salmonella 4,[5],12:i:-) lacks expression of the second flagellar phase of $S$. Typhimurium, which has the antigenic formula 4,[5],12:i:1,2 [5]. Salmonella 4,[5],12:i:- has the $\mathrm{O} 4$ antigen, B serogroup and $\mathrm{i}$ antigen, but lacks the 1,2 antigen. Flagellar phase variation is induced by inversion of the genetic region called the $\mathrm{H}$ segment, which contains the hin gene encoding DNA invertase and a promoter for the $f j B$ gene. The $f j B$ gene constitutes an operon with the $f j A$ gene [5]. Therefore, $S$. Typhimurium variants result from alterations in the expression of $f l j A, f j B$ and $h i n$ genes $[16,19]$. The European Food Safety Authority has suggested a polymerase chain reaction (PCR) protocol

*Correspondence to: Yang, D.-K., Viral Disease Division, Animal and Plant Quarantine Agency, Anyang, Gyeonggi-do 430-757, MAFRA, Republic of Korea. e-mail: yangdk@korea.kr

(C)2015 The Japanese Society of Veterinary Science

This is an open-access article distributed under the terms of the Creative Commons Attribution Non-Commercial No Derivatives (by-nc-nd) License $<$ http://creativecommons.org/licenses/by-nc-nd/3.0/>. supporting traditional slide agglutination serotyping to clearly differentiate Salmonella 4,[5],12:i:- from $S$. Typhimurium [5]. Since Salmonella 4,[5],12:i:- was first isolated in Spain [9], the serovar has been considered to be the predominant agent of food poisoning in Europe and the United States over the last 10 years [16]. In Korea, the first outbreak of food poisoning caused by Salmonella 4,[5],12:i:- was reported in 2001, and Salmonella 4,[5],12:i:- was isolated from poultry in 2009-2011 [21, 22]. However, there are no reports of Salmonella 4,[5],12:i:- isolates from cattle or pigs in Korea.

Epidemiological studies of $S$. Typhimurium have been carried out using classical methods, such as phage typing and antimicrobial resistance testing and molecular genetic methods, such as multiple-locus variable-number tandem repeat analysis (MLVA) and pulsed-field gel electrophoresis (PFGE) [2, 6, 14, $18,23,28]$. Among these tools, MLVA is based on multiplex PCR amplification of VNTR (variable-number tandem repeat) loci followed by sizing of the products using capillary electrophoresis. The method is highly discriminatory, and, unlike PFGE, it is easy to perform, robust and rapid [12, 13, 20].

In this study, we examined the prevalence of $S$. Typhimurium in pigs presenting with diarrhea in South Korea, and we isolated Salmonella 4,[5],12:i- from farmed pigs for the first time. In addition, phage typing, antimicrobial resistance tests, flagellar gene distribution analyses and MLVAs were used to compare the characteristics of $S$. Typhimurium and Salmonella 4,[5],12:i:-.

Porcine fecal samples were collected from livestock presenting with digestive diseases, such as diarrhea and enteritis, by Choong-Ang Vaccine Laboratories. A total of 896 fecal samples were obtained from nine provinces (Gyeonggi, Gangwon, Chungbuk, Chungnam, Jeonbuk, Jeonnam, Gyeongbuk, Gyeongnam and Jeju) throughout 
Table 1. List of the oligonucleotide primers used for PCR of Salmonella

\begin{tabular}{|c|c|c|c|c|}
\hline Gene & Function & Nucleotide sequence $\left(5^{\prime}-3^{\prime}\right)$ & $\begin{array}{l}\text { Size of amplicon } \\
\text { (bp) }\end{array}$ & Ref. \\
\hline $\begin{array}{l}\text { STM } \\
0292\end{array}$ & Putative RHS-family protein & $\begin{array}{l}\text { ATGCGGGTATGACAAACCCT } \\
\text { TTAGCCCCATTTGGACCTTT }\end{array}$ & 94 & {$[1]$} \\
\hline $\begin{array}{l}\text { STM } \\
2235\end{array}$ & Putative phage protein & $\begin{array}{l}\text { CAGACCAGGTAAGTTTCTGG } \\
\text { CGCATATTTGGTGCAGAAAT }\end{array}$ & 196 & {$[1]$} \\
\hline $\begin{array}{l}\text { STM } \\
4493\end{array}$ & Putative cytoplasmic protein & $\begin{array}{l}\text { TTTACCTCAATGGCGGAACC } \\
\text { CCCAAAAGCTGGGTTAGCAA }\end{array}$ & 303 & {$[1]$} \\
\hline $\begin{array}{c}\text { STM } \\
1053-1997\end{array}$ & - & $\begin{array}{l}\text { CCATTTTTATACTGCCAGTCGCC } \\
\text { CAGCGAAATACTGATGGCGG }\end{array}$ & 614 & {$[27]$} \\
\hline $\begin{array}{l}\text { STM } \\
2740\end{array}$ & Integrase, phage family & $\begin{array}{l}\text { AATGTGGAGATCGCTGGCGCG } \\
\text { AGTTCGCCGCCGAACCCC }\end{array}$ & 980 & {$[27]$} \\
\hline $\begin{array}{l}\text { STM } \\
2757\end{array}$ & Putative cytoplasmic protein & $\begin{array}{l}\text { ATGATGATGGCGTAATGGCGC } \\
\text { AAAACGTTCCGGTGCGGCG }\end{array}$ & 717 & {$[27]$} \\
\hline $\begin{array}{l}\text { STM } \\
2773\end{array}$ & Glucosyltransferase homolog & $\begin{array}{l}\text { TTCGATTCGGAAGCGGGTTATCGCCG } \\
\text { CTCGCGAAGCGCGCG }\end{array}$ & 858 & {$[27]$} \\
\hline$f j A$ & Repressor of phase 1 Flagellin gene & $\begin{array}{l}\text { TTCATTAGGTCCCCTCCGG } \\
\text { ATTCAGCCCCGTGAATTCGGG }\end{array}$ & 642 & {$[27]$} \\
\hline$f j B$ & Phase 2 flagellin structural protein & $\begin{array}{l}\text { TTTACCGTCTACGCCACCC } \\
\text { GGTACTACACTGGATGTATCGGG }\end{array}$ & 561 & {$[27]$} \\
\hline hin & $\begin{array}{l}\mathrm{H} \text { inversion: regulation of flagellar } \\
\text { gene expression }\end{array}$ & $\begin{array}{l}\text { TGGCTACTATTGGGTATATTCGGG } \\
\text { CTCGCGAAGCGCGCG }\end{array}$ & 570 & {$[27]$} \\
\hline
\end{tabular}

the Republic of Korea from 2011 to 2012. All samples were collected under aseptic conditions, mixed with $45 \mathrm{ml}$ of buffered peptone water and incubated for $20 \mathrm{hr}$ at $37^{\circ} \mathrm{C}$. After incubation, $0.1 \mathrm{~m} l$ of each sample was inoculated into $10 \mathrm{~m} l$ of Rappaport Vassiliadis (RV) R10 broth (Merck, Darmstadt, Germany) and then incubated for $24 \mathrm{hr}$ at $42^{\circ} \mathrm{C}$. One loop of $\mathrm{RV}$ culture was streaked onto the surface of both xylose lysine deoxycholate agar (Difco, Becton, Dickinson, and Co., Sparks, MD, U.S.A.) and Salmonella Shigella agar (Difco, Becton, Dickinson, and Co.) plates, and then, suspected colonies were serotyped using Salmonella antisera (Denka Seiken, Tokyo, Japan) according to the method of Ewing [11]. Phage types were determined using $S$. Typhimurium phages obtained from Public Health England public health laboratories (Colindale, London, U.K.). Multiplex PCR was performed to detect the $S$. Typhimurium-related genes STM0292, STM2235 and STM4493 (Table 1) [1]. Isolates of $S$. Typhimurium were deposited in the Korea Veterinary Culture Collection (KVCC), where they were stored until further use.

Minimal inhibitory concentrations were determined by broth microdilution using the Sensititre microdilution panel by Thermofisher Inc. (formerly TREK Diagnostic Systems; Cleveland, OH, U.S.A.). The tested antimicrobial agents were ampicillin, amoxicillin/clavulanic acid, cefoxitin, ceftiofur, cephalothin, chloramphenicol, ciprofloxacin, colistin, florfenicol, gentamicin, nalidixic acid, neomycin, streptomycin, tetracycline and trimethoprim-sulfamethoxazole. Susceptibility was determined in accordance with the guidelines of the Clinical and Laboratory Standards Institute [8]. Escherichia coli ATCC 25922 was used as a control strain.

The expression of flagellar genes (STM1053-1997, STM2740, STM2757, STM2773, fljA, $f j B$ and hin) was as- sessed by PCR (Table 1) as described previously [27]. DNA fragments were separated on a $1.5 \%$ agarose gel. Fragments of the appropriate size were extracted from the gel and purified using a Gel Extraction Kit (Qiagen Inc., Valencia, CA, U.S.A.), followed by sequencing. A database search was performed using the BLAST program at the National Center for Biotechnology Information (http://www.ncbi.nlm.nih.gov).

MLVA was performed following the standardized procedure established by pulseNet [3]. The profiles of the isolates were compared using BioNumerics software (Applied Maths, Sint-Martens-Latem, Belgium) with the categorical coefficient and unweighted-pair group method with average linkages.

Salmonella spp. were tried to be isolated from nine provinces between 2011 and 2012. Fifty-nine Salmonella spp. out of 896 diarrheic pigs samples were isolated. Out of these isolates, 50 were identified as $S$. Typhimurium. In total, $S$. Typhimurium was isolated from $7.19 \%$ (21/292) of the pigs examined in 2011 and $4.8 \%(29 / 604)$ of the pigs examined in 2012. The total numbers of $S$. Typhimurium isolates from each region during the study period were as follows: Chungnam $(\mathrm{n}=15)$, Gyeongbuk $(\mathrm{n}=9)$, Chungbuk $(\mathrm{n}=6)$, Gyeongnam $(\mathrm{n}=5)$, Gyeonggi $(\mathrm{n}=4)$, Jeonbuk $(\mathrm{n}=4)$, Jeju $(n=2)$, Jeonnam $(n=1)$, Gangwon $(n=0)$ and unknown $(n=4)$. The seasonal frequencies of $S$. Typhimurium incidences on pig farms in 2011-2012 were $0.0-10.0 \%$ from January to March, $2.0-20.0 \%$ from April to June, $6.0-14.0 \%$ from July to September and $6.0-16.0 \%$ from October to December. The phage typing results for the $S$. Typhimurium isolates in this study were as follows: U288, 6 strains; U302, 3 strains; DT193, 3 strains; U310, 1 strain; U291, 1 strain; and reacted, but did not conform (RDNC), 36 strains. Among the S. Typhimurium isolates, four strains (KVCC-BA1300254, KVCC-BA1300255, KVCC-BA1400078 and KVCC- 


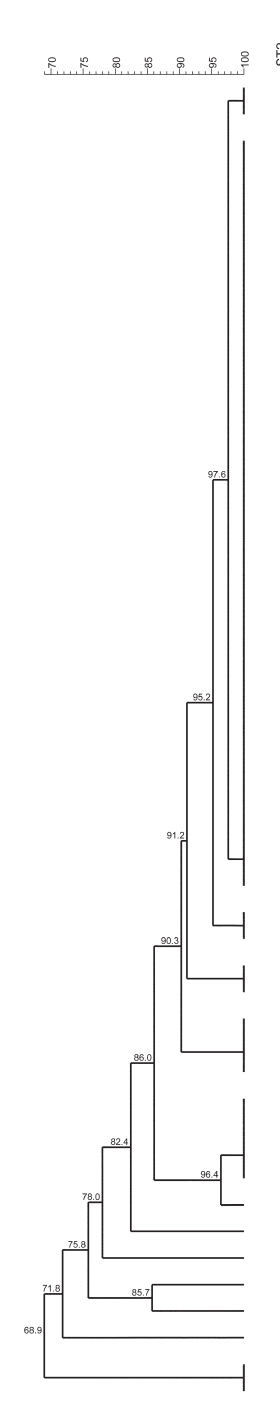

\begin{tabular}{|c|c|c|c|c|c|c|c|}
\hline & $\stackrel{\circ}{\infty}$ & $\stackrel{\infty}{\infty}$ & 第 & $\frac{2}{6}$ & 点 & E & KVCC No \\
\hline & 15 & 33 & 4 & 13 & 5 & 28 & KVCC-BA1300261 \\
\hline & 15 & 33 & 4 & 13 & 5 & 28 & KVCC-BA1300267 \\
\hline & 12 & 33 & 4 & 11 & 5 & 28 & KVCC-BA1300152 \\
\hline & 14 & 33 & 4 & 10 & 5 & 28 & KVCC-BA1300156 \\
\hline 22 & 13 & 33 & 4 & 10 & 5 & 28 & KVCC-BA1300157 \\
\hline 22 & 14 & 33 & 4 & 10 & 5 & 28 & KVCC-BA1300159 \\
\hline 22 & 14 & 33 & 4 & 11 & 5 & 28 & KVCC-BA1300160 \\
\hline 22 & 14 & 33 & 4 & 10 & 5 & 28 & KVCC-BA1300162 \\
\hline 22 & 14 & 33 & 4 & 11 & 5 & 28 & KVCC-BA1300165 \\
\hline 22 & 12 & 33 & 4 & 10 & 5 & 28 & KVCC-BA1300166 \\
\hline 22 & 13 & 33 & 4 & 10 & 5 & 28 & KVCC-BA1300167 \\
\hline 22 & 15 & 33 & 4 & 8 & 5 & 28 & KVCC-BA1300256 \\
\hline 22 & 14 & 33 & 4 & 8 & 5 & 28 & KVCC-BA1300257 \\
\hline 22 & 14 & 33 & 4 & 11 & 5 & 28 & KVCC-BA1300260 \\
\hline 22 & 15 & 33 & 4 & 10 & 5 & 28 & KVCC-BA1300262 \\
\hline 22 & 13 & 33 & 4 & 10 & 5 & 28 & BA1300263 \\
\hline 22 & 13 & 33 & 4 & 10 & 5 & 28 & KVCC-BA1300264 \\
\hline 22 & 15 & 33 & 4 & 9 & 6 & 28 & KVCC-BA1300266 \\
\hline 22 & 15 & 33 & 4 & 11 & 5 & 28 & KVCC-BA1300269 \\
\hline 22 & 14 & 33 & 2 & 11 & 5 & 28 & KVCC-BA1300274 \\
\hline 22 & 14 & 33 & 2 & 11 & 5 & 28 & KVCC-BA1300275 \\
\hline 22 & 14 & 33 & 4 & 11 & 5 & 28 & KVCC-BA1400066 \\
\hline 22 & 14 & 33 & 4 & 11 & 6 & 28 & KVCC-BA1400067 \\
\hline 22 & 15 & 33 & 4 & 11 & 5 & 28 & KVCC-BA1400069 \\
\hline 22 & 13 & 33 & 4 & 10 & 5 & 28 & KVCC-BA1400070 \\
\hline 22 & 14 & 33 & 4 & 11 & 6 & 28 & KVCC-BA1400071 \\
\hline 22 & 14 & 33 & 4 & 9 & 5 & 28 & KVCC-BA1400073 \\
\hline 22 & 14 & 33 & 4 & 9 & 5 & 28 & KVCC-BA1400074 \\
\hline 22 & 15 & 33 & 4 & 11 & 5 & 28 & KVCC-BA1400076 \\
\hline 22 & 14 & 33 & 4 & 11 & 5 & 28 & KVCC-BA1400077 \\
\hline 22 & 14 & 33 & 4 & 10 & 5 & 28 & KVCC-BA1400079 \\
\hline 22 & 16 & 33 & 4 & 7 & 5 & 28 & KVCC-BA1300172 \\
\hline 22 & 16 & 33 & 4 & 9 & 5 & 28 & KVCC-BA1300270 \\
\hline 22 & 13 & 33 & 4 & 7 & 5 & 28 & KVCC-BA1300154 \\
\hline 22 & 10 & 33 & 4 & 10 & 3 & 28 & KVCC-BA1400080* \\
\hline 22 & 10 & 33 & 4 & 12 & 4 & 28 & KVCC-BA1300259 \\
\hline 22 & 12 & 33 & 4 & 12 & 5 & 28 & KVCC-BA1300273 \\
\hline 22 & 9 & 33 & 4 & 12 & 5 & 28 & KVCC-BA1300272 \\
\hline 22 & 14 & 33 & 4 & 16 & 6 & 28 & KVCC-BA1300155 \\
\hline 22 & 14 & 33 & 4 & 16 & 6 & 28 & KVCC-BA1300164 \\
\hline 22 & 11 & 36 & 4 & 14 & 3 & 28 & KVCC-BA1300254* \\
\hline 22 & 13 & 33 & 4 & 16 & 3 & 28 & KVCC-BA1400072 \\
\hline 22 & 14 & 33 & 4 & 18 & 6 & 28 & KVCC-BA1400075 \\
\hline 22 & 15 & 33 & 4 & 11 & 5 & 6 & KVCC-BA1300258 \\
\hline 22 & 17 & 19 & 4 & 13 & 5 & 28 & KVCC-BA1400068 \\
\hline 22 & 14 & 33 & 4 & 14 & 5 & 10 & KVCC-BA1300170 \\
\hline 22 & 14 & 33 & 4 & 15 & 5 & 24 & KVCC-BA1300271 \\
\hline 22 & 10 & 27 & 7 & 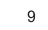 & 3 & 28 & KVCC-BA1400078* \\
\hline 25 & 8 & 36 & & 8 & 2 & 8 & KVCC-BA1300255* \\
\hline & 8 & 33 & & 10 & & 10 & KVCC-BA1300265 \\
\hline
\end{tabular}

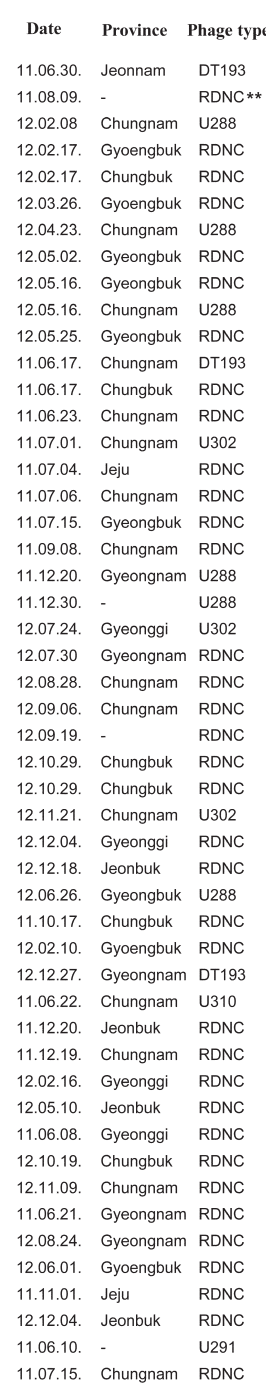

\begin{tabular}{|c|c|}
\hline Antimicrobial resistance pattern $* * *$ & Cluster \\
\hline AMP/CHL/NAL/STR/SXT/TET & \\
\hline NAL/STR/TET & \\
\hline AMP/GEN/TET & \\
\hline AMP/CHL/GEN/STR/TET & \\
\hline AMP/GEN/TET & \\
\hline AMP/CHL/GEN/NAL/STR/SXT/TET & \\
\hline AMP/CHL/GEN/NAL/STR/TET & \\
\hline AMP/GEN/NAL/STR/TET & \\
\hline AMP/CHL/GEN/NAL/STR/SXT/TET & \\
\hline AMP/CHL/GEN/NAL/STR/TET & \\
\hline AMP/GEN/TET & \\
\hline AMP/CHL/GEN/NAL/STR/TET & \\
\hline AMP/CEP/CHL/GEN/NAL/STR/SXT & \\
\hline AMP/GEN/NAL/STR/SXT/TET & \\
\hline AMP/CHL/GEN/NAL/STR/SXT/TET & \\
\hline AMP/CHL/GEN/NAL/STR/SXT/TET & \\
\hline AMP/GEN/NAL/STR & \\
\hline AMP/CHL/GEN/NAL/STR/SXT/TET & \\
\hline CHL/GEN/NAL/STR/TET & \\
\hline NAL/STR/TET & \\
\hline NAL/STR/TET & \\
\hline AMP/CHL/FOX/NAL/STR/TET & \\
\hline AMP/CHL/GEN/NAL/STR/SXT/TET & $A(n=43)$ \\
\hline CHL/NAL/STR/SXT/TET & \\
\hline AMP/GEN/TET & \\
\hline AMP/CHL/GEN/NAL/STR/SXT & \\
\hline AMP/CHL/GEN/NAL/STR/SXT & \\
\hline AMP/CHL/GEN/NAL/STR/SXT & \\
\hline CHL/GEN/NAL/STR/SXT & \\
\hline AMP/CHL/NAL/STR/SXT & \\
\hline AMP/CHL/GEN/NAL/STR/SXT/TET & \\
\hline CHL/NAL/STR/TET & \\
\hline AMP/CHL/GEN/NAL/STR/TET & \\
\hline AMP/GEN/TET & \\
\hline AMP/STR/TET & \\
\hline AMP/AUG/CEP/CHL/GEN/NAL/STR/SXT/TET & \\
\hline AMP/CHL/GEN/NAL/STR/TET & \\
\hline AMP/GEN/TET & \\
\hline AMP/CEP/CHL/NAL/STR/TET & \\
\hline AMP/CHL/STR/TET & \\
\hline STR & \\
\hline AMP/CHL/GEN/NAL/STR/SXT & \\
\hline AMP/CHL/STR/TET & \\
\hline NAL/STR/SXT/TET & $B(n=1)$ \\
\hline AMP/NAL & $\mathrm{C}(\mathrm{n}=1)$ \\
\hline AMP/CHL/GEN/STR/TET & $D(n=2)$ \\
\hline AMP/AUG/CEP/CHL/STR/SXT/TET & $D(n=2)$ \\
\hline AMP/STR/TET & $E(n=1)$ \\
\hline- & $F(n=2)$ \\
\hline AMP/CEP/CHL/GEN/NAL/STR/TE & \\
\hline
\end{tabular}

Fig. 1. MLVA profiles of $S$. Typhimurium that were analyzed using the BioNumerics program (Applied Maths) to generate a dendrogram based on the Dice coefficient. Fifty strains were divided into six clusters at similarity rates of $85 \%$. *Salmenella 4,5,12:i:- **RDNC: reacted, but did not conform. ***AMP, ampicillin; CEP, cephalothin; CHL, chloramphenicol; GEN, gentamicin; NAL, nalidixic acid; STR, streptomycin; SXT, trimethoprim/sulfamethoxazole; TET, tetracycline

BA1400080) were identified as Salmonella 4,[5],12:i:-, and had the $\mathrm{O} 4$ antigen, B serogroup and i antigen, but lacked the 1,2 antigen. The phage types of the four Salmonella 4,[5],12:i:- strains were U291, DT193 and RDNC (Fig. 1).

The antimicrobial resistance patterns of the $50 \mathrm{~S}$. Typhimurium isolates are presented in Fig. 1. Of the 50 isolates, 47 were resistant to three antimicrobial agents in different classes, and 15 of these 47 isolates were pentaresistant to ampicillin, chloramphenicol, streptomycin, tetracycline and nalidixic acid. The higher level of antimicrobial resistance was observed among $S$. Typhimurium isolates from diarrheic pigs. Among the Salmonella 4,[5],12:i:- isolates, both KVCC-BA1400078 and KVCC-BA1400080 were resistant to ampicillin, streptomycin and tetracycline.

The flagellar gene expression data are shown in Table 2.
All flagellar genes were present in 46 of the $S$. Typhimurium isolates (data not shown). However, the four Salmonella 4,[5],12:i:- isolates had different flagellar genes. One strain (KVCC-BA1400078) lacked all three flagellar genes ( $f j A$, $f j B$ and hinA) and three strains (KVCC-BA1300254, KVCC-BA1300255 and KVCC-BA1400080) had hin, but not $f j A$ and $f j B$.

Figure 1 shows the MLVA profiles of the $50 S$. Typhimurium isolates. The isolates were classified into 1 major cluster (A) and 5 minor clusters (B, C, D, E and F) based on $85 \%$ similarity in the MLVA, as follows: cluster A, 43 isolates (Chungnam: 14; Gyeongbuk: 8; Chungbuk: 6; Gyeonggi: 4; Gyeongnam: 3; Jeonbuk: 3; Jeonnam: 1; Jeju: 1; and unknown: 3); cluster B, 1 isolate (Gyeongnam); cluster C, 1 isolate (Gyeongnam); cluster D, 2 isolates (Gyeongbuk: 1; 
Table 2. Distribution of flagella gene in Salmonella I 4,[5],12:i:-

\begin{tabular}{|c|c|c|c|c|c|c|c|c|c|}
\hline \multirow[b]{2}{*}{ KVCC No. } & \multirow[b]{2}{*}{ Date } & \multirow[b]{2}{*}{ Province } & \multicolumn{7}{|c|}{ Gene of Salmonella } \\
\hline & & & $\begin{array}{l}\text { STM } \\
2740\end{array}$ & $\begin{array}{l}\text { STM } \\
2757\end{array}$ & $\begin{array}{c}\text { STM } \\
1053-1997\end{array}$ & $\begin{array}{l}\text { STM } \\
2773\end{array}$ & $f j A$ & $f j B$ & hin \\
\hline KVCC-BA1300254 & 2011.06. & Gyeonggi & + & + & + & - & - & - & + \\
\hline KVCC-BA1300255 & 2011.06 & - & - & - & + & - & - & - & + \\
\hline KVCC-BA1400078 & 2012.12 & Jeonbuk & + & + & - & + & - & - & - \\
\hline KVCC-BA1400080 & 2012.12 & Gyeongnam & + & + & - & + & - & - & + \\
\hline
\end{tabular}

and Jeju: 1); cluster E, 1 isolate (Jeonbuk); and cluster F, 2 isolates (Chungnam: 1; unknown: 1). In this study, most of the $S$. Typhimurium isolates from Korean pigs were classified into cluster A, even though they were isolated from various locations nationwide. Among the Salmonella 4,[5],12:i:isolates, KVCC-BA1300254 and KVCC-BA1400080 were classified into cluster $\mathrm{A}$, while the remaining two strains, KVCC-BA1400078 and KVCC-BA1300255, were classified into clusters $\mathrm{E}$ and $\mathrm{F}$, respectively.

In Korea, $S$. Typhimurium is well known as a causative agent of diarrhea in pigs [26]. $S$. Typhimurium was isolated from $13.9 \%(42 / 302)$ of the pigs examined during 2000-2006 and $22.3 \%(96 / 431)$ of the pigs examined during 2006-2007 [14, 25]. In this study, the prevalence of $S$. Typhimurium $(7.19 \%$ in 2011 and $4.80 \%$ in 2012) was slightly lower than that of $S$. Typhimurium reported previously $[14,25]$. However, recently, there have been many reports of $S$. Typhimurium variants isolated from humans and warm-blooded animals worldwide. Salmonella 4,[5],12:i:- is a variant of $S$. Typhimurium and an important pathogenic agent of food poisoning. According to the results of a surveillance project for Salmonella isolated from a pig slaughterhouse in Europe, Salmonella 4,[5],12:i:- has been increasing exponentially in Germany [10]. A recent report from the United States indicated that Salmonella 4,[5],12:i:occurrences have increased exponentially in humans [7]. As a result of tracing Salmonella 4,[5],12:i:- in Korea, the serovar was isolated from humans in 2001 and from poultry in 2009-2011 [21, 22]. However, there were no reported isolates from pigs, despite much investigation. In this study, four strains of Salmonella 4,[5],12:i:- were isolated from 896 pigs between 2011 and 2012. Expression of the $f j A B$ operon (flj $\mathrm{AljB}$ and hin) in Salmonella 4,[5],12:i:- varies from country to country [27]. The four Salmonella 4,[5],12:i:strains isolated in this study exhibited flagellar gene profiles that have not yet been reported in Korea. One strain in particular, KVCC-BA1400078, had high identity to a Salmonella 4,[5],12:i:- clone isolated from a human in the United States in the PCR screens to contain flagella gene [27]. It is difficult to determine whether Salmonella 4,[5],12:i:- existed previously in swine in Korea or was brought in from outside the country; however, the emergence of Salmonella 4,[5],12:i:- isolates in Korean pigs suggests an urgent need for the establishment of a strict Hazard Analysis and Critical Control Point program in the process of importing animals and farming animals.

More than $90 \%$ of the isolates obtained in 2006-2007 exhibited antimicrobial resistance to streptomycin, tetracycline and sulfamethoxazole [25]. To avoid the misuse of antimicrobial agents in livestock and protect public health through safe management, the use of antimicrobial agents in livestock decreased steadily, beginning in July 2011, until it was finally banned in March 2013 in accordance with Korean policy. Compared to previous studies, the prevalence of antimicrobial resistance decreased slightly in this study $[24,25,27]$. In terms of the distribution of phage types among Salmonella 4,[5],12:i:- isolates from several European countries, most strains are of phage type DT193 or DT120, and these strains are multidrug-resistant [13]. In Korea, almost all $S$. Typhimurium and Salmonella 4,[5],12:i:- isolates exhibited ampicillin, streptomycin and tetracycline resistance. In particular, Salmonella 4,[5],12:i:- strain KVCCBA1400080 (phage type DT193) was resistant to ampicillin, streptomycin and tetracycline, similar to phage type DT193 strains isolated in Europe [13]. Accordingly, antimicrobial susceptibility monitoring of Salmonella isolates from pigs is needed for appropriate prevention and treatment.

MLVA exhibited sufficient discriminatory power in both Brucella isolates from cattle and $S$. Gallinarum isolates from chickens in Korea [15, 17]. However, by MLVA alone, Salmonella 4,[5],12:i:- cannot be distinguished from the $S$. Typhimurium cluster, because of the close genetic relationship between the two serovars. However, profiles created by MLVA further classified the causative agents from each outbreak.

In conclusion, $S$. Typhimurium is commonly isolated from pigs presenting with diarrhea, and this is the first reported detection of Salmonella 4,[5],12:i:- from pigs in Korea. The isolation of Salmonella 4,[5],12:i:- is increasing worldwide, and the number of antimicrobial-resistant strains is increasing steadily. Therefore, continuous monitoring at the national level is necessary for food poisoning tracking and pig disease analysis.

ACKNOWLEDGMENT. This study was supported by a grant (N-AD20-2010-19-01) from the Animal and Plant Quarantine Agency, Ministry of Agriculture, Food and Rural Affairs (MAFRA), Republic of Korea.

\section{REFERENCES}

1. Akiba, M., Kusumoto, M. and Iwata, T. 2011. Rapid identification of Salmonella enterica serovars, Typhimurium, Choleraesuis, Infantis, Hadar, Enteritidis, Dublin and Gallinarum, 
by multiplex PCR. J. Microbiol. Methods 85: 9-15. [Medline] [CrossRef]

2. Amavisit, P., Boonyawiwat, W. and Bangtrakulnont, A. 2005. Characterization of Salmonella enterica serovar Typhimurium and monophasic Salmonella serovar 1,4,[5],12:i:- isolates in Thailland. J. Clin. Microbiol. 43: 2736-2740. [Medline] [CrossRef]

3. Anonymous. 2007. Laboratory standard operating procedure for PulseNet MLVA of Salmonella enteric serotype TyphimuriumBeckman Coulter CEQ 8000 platform. http://www.pulsenetinternational.org/SiteCollectionDocuments/mlva/PNL21_MLVA_ 20Salm_20T_20Beckman_20Protocol.pdf.

4. Asai, T., Otagiri, Y., Osumi, T., Namimatsu, T. and Sato, S. 2002. Isolation of Salmonella from diarrheic feces of pigs. J. Vet. Med. Sci. 64: 159-160. [Medline] [CrossRef]

5. Barco, L., Longo, A., Lettini, A. A., Cortini, E., Saccardin, C., Minorello, C., Olsen, J. E. and Ricci, A. 2014. Molecular characterization of "inconsistent" variants of Salmonella Typhimurium isolated in Italy. Foodborne Pathog. Dis. 11: 497-499. [Medline] [CrossRef]

6. Bruun, T., Sorensen, G., Forshell, L. P., Jensen, T., Nygarg, K., Kapperud, G., Lindstedt, B. A., Berglund, T., Wingstrard, A., Petersen, R. F., Muller, L., Kjelso, C., Lvarsson, S., Hjertqvist, M., Lofdahl, S. and Ethelberg, S. 2009. An outbreak of Salmonella Typhimurium infections in Denmark, Norway and Sweden. 2008. Euro Surveill. 14: 1-6. [Medline]

7. Centers for Disease Control and Prevention. 2008. Salmonella surveillance: annual summary. 2006.

8. Clinical and Laboratory Standards Institute 2014. Performance Standards for Antimicrobial Susceptibility Testing; 24th Informational Supplement. M100-S24. Clinical and Laboratory Standards Institutes, Wayne.

9. Echeita, M. A., Aladuena, A., Cruchaga, S. and Usera, M. A. 1999. Emergence and spread of an atypical Salmonella enteric susp.enterica serotype 4,5,12:i:- strain in Spain. J. Clin. Microbiol. 37: 3425. [Medline]

10. European Food Safety Authority 2008. Report of the task force on zoonoses data collection on the analysis of the baseline survey on the prevalence of Salmonella in slaughter pigs, in the EU, 2006-2007. EFSA Journal 135: 1-111.

11. Ewing, W. H. 1986. Serologic identification of Salmonella. pp.201-238. In: Edwards and Ewing's identification of Enterobacteriaceae, 4th ed. (Ewing, W. H. ed.), Elsevier, New York.

12. Hernandez, S. M., Keel, K., Sanchez, S., Trees, E., Gerner-smidi, P., Adams, J. K., Cheng, Y., Ray, A. 3rd., Martin, G., Presotto, A., Ruder, M. G., Brown, J., Blehert, D. S., Cottrell, W. and Maurer, J. J. 2012. Epidermiology of Salmonella enterica subsp. enterica Serovar Typhimurium strain associated with a songbird outbreak. Appl. Environ. Microbiol. 78: 7290-7298. [Medline] [CrossRef]

13. Hopkins, K. L., Kirchner, M., Guerra, B., Granier, S. A., Lucarelli, C., Porrero, M. C., Jakubczak, A., Threlfall, E. J. and Mevius, D. J. 2010. Multiresistant Salmonella enterica serovar 4,[5],12:i:- in Europe: a new pandemic strains. Euro Surveill. 15: 19580. [Medline]

14. Hur, J., Choi, Y. Y., Park, J. H., Jeon, B. W., Lee, H. S., Kim, A. R. and Lee, J. H. 2011. Antimicrobial resistance, virulenceassociated genes, and pulsed-field gel electrophoresis profiles of Salmonella enterica subsp. enterica serovar Typhimurium isolated from piglets with diarrhea in Korea. Can. J. Vet. Res. 75: 49-56. [Medline]

15. Her, M., Kang, S. I., Cho, D. H., Cho, Y. S., Hwang, I. Y., Heo, Y. R., Jung, S. C. and Yoo, H. S. 2009. Application and evaluation of the MLVA typing assay for the Brucella abortus strains isolated in Korea. BMC Microbiol. 9: 230. [Medline] [CrossRef]
16. Ido, N., Lee, K., Iwabuchi, K., Izumiya, H., Uchida, I., Kusumoto, M., Iwata, T., Ohnishi, M. and Akiba, M. 2014. Characteristics of Salmonella enterica serovar 4,[5],12:i:- as a monophasic variant of serovar Typhimurium. PLOS ONE 9: e104380. [Medline]

17. Kang, M. S., Kwon, Y. K., Oh, J. Y., Call, D. R., An, B. K., Song, E. A., Kim, J. Y., Shin, E. G., Kim, M. J., Kwon, J. H. and Chung, G. S. 2011. Multilocus variable-number tandem repeat analysis for subtyping Salmonella enterica serovar Gallinarum. Avian Pathol. 40: 559-564. [Medline] [CrossRef]

18. Kim, J., Hyeon, J. Y., Lee, E., Lee, D., Kim, Y. J., Kim, Y. J. and Kim, S. 2014. Molecular epidemiological analysis of five outbreaks associated with Salmonella enterica serovar Enteritidis between 2008 and 2010 on Jeju island, Republic of Korea. Foodborne Pathog. Dis. 11: 38-42. [Medline] [CrossRef]

19. Kim, S. H., Kim, S., Lee, S. W., Kang, Y. H. and Lee, B. 2005. Rapid serological identification for monophasic Salmonella serovars with a hin gene-specific polymerase chain reaction. $J$. Bac. Virol. 35: 291-297.

20. Kurosawa, A., Imamura, T., Tanaka, K., Tamamura, Y., Uchida, I., Kobayashi, A., Hata, E., Kanno, T., Akiba, M., Yukawa, S. and Tamura, Y. 2012. Molecular typing of Salmonella enterica serotype Typhimurium and serotype 4,5,12:- isolates from cattle by multiple-locus variable-number tandem-repeats analysis. Vet. Microbiol. 160: 264-268. [Medline] [CrossRef]

21. Lee, D. Y., Lee, E., Min, J. E., Kim, S. H., Oh, H. B. and Park, M. S. 2011. Epidemic by Salmonella I 4, [5],12:i:- and characteristics of isolates in Korea. Infect. Chemother. 43: 186-190. [CrossRef]

22. Lee, D. Y., Kang, M. S., Kwon, Y. K., An, B. K., Kim, Y. J., Heo, E. J., Moon, J. S., Lee, E. and Park, H. 2012. First isolation of Salmonella I 4,[5],12:i:-from domestic animals in Korea. Korean J. Vet. Res 52: 285-288.

23. Lee, K. E. and Lee, Y. H. 2007. Isolation of multidrug-resistant Salmonella Typhimurium DT104 from swine in Korea. J. Microbiol. 45: 590-592. [Medline]

24. Lee, K. E., Jung, J. H., Jung, B. Y., Park, Y. H. and Lee, Y. H. 2011. Characterization of nalidixic acid resistant and Fluoroquinolone reduced susceptible Salmonella Typhimurium in swine. J. Food Prot. 74: 610-615. [Medline] [CrossRef]

25. Lim, S. K., Lee, H. S., Nam, H. M., Jung, S. C., Koh, H. B. and Roh, I. S. 2009. Antimicrobial resistance and phage types of Salmonella isolates from healthy and diarrheic pigs in Korea. Foodborne Pathog. Dis. 6: 981-987. [Medline] [CrossRef]

26. Rayamajhi, N., Kang, S. G., Kang, M. L., Lee, H. S., Park, K. Y. and Yoo, H. S. 2008. Assessment of antibiotic resistance phenotype and integrons in Salmonella enterica serovar Typhimurium isolated from Swine. J. Vet. Med. Sci. 70: 1133-1137. [Medline] [CrossRef]

27. Soyer, Y., Mereno Switt, A., Davis, M. A., Maurer, J., McDonough, P. L., Schoonmaker-Bopp, D. J., Damas, N. B., Root, T., Warnick, L. D., Grohn, Y. T. and Wiedmann, M. 2009. Salmonella enteric serotype 4,5,12:i:-, an emerging Salmonella serotype that represents multiple distinct clones. J. Clin. Microbiol. 47: 3546-3556. [Medline] [CrossRef]

28. Tamamura, Y., Uchida, I., Tanaka, K., Okazaki, H., Tezuka, S., Hanyu, H., Kataoka, N., Makino, S., Kishima, M., Kubota, T., Kanno, T., Hatama, S., Ishihara, R., Hata, E., Yamada, H., Nakaoka, Y. and Akiba, M. 2011. Molecular epidemiology of Salmonella enterica serovar Typhimurium isolates from cattle in Hokkaido, Japan: Evidence of clonal replacement and characterization of the disseminated clone. Appl. Environ. Microbiol. 77: 1739-1750. [Medline] [CrossRef] 